\title{
Une télévision satellite suisse? Libéralisation de l'audiovisuel public et «nouveaux médias»: l'année 1980
}

\section{Marie Sandoz}

\section{(2) OpenEdition Journals}

Édition électronique

URL : http://journals.openedition.org/edl/2347

DOI : $10.4000 /$ edl.2347

ISSN : 2296-5084

Éditeur

Université de Lausanne

\section{Édition imprimée}

Date de publication : 15 mars 2020

Pagination : 59-62

ISBN : 978-2-940331-73-4

ISSN : 0014-2026

Référence électronique

Marie Sandoz, « Une télévision satellite suisse? Libéralisation de l'audiovisuel public et «nouveaux médias»: l'année 1980 », Études de lettres [En ligne], 312 | 2020, mis en ligne le 24 mars 2020, consulté le 16 septembre 2020. URL : http://journals.openedition.org/edl/2347 ; DOI : https://doi.org/10.4000/ edl. 2347 


\section{UNE TÉLÉVISION SATELLITE SUISSE? LIBÉRALISATION DE L'AUDIOVISUEL PUBLIC ET «NOUVEAUX MÉDIAS»: L'ANNÉE 1980}

Le 14 mai 1980, quatorze éditeurs de presse et agences publicitaires suisses se réunissent à Bâle pour fonder l'entreprise Tel-Sat. Le sommet du conseil d'administration se compose de Jean-Claude Nicole, Josef Jäger et Fritz Latscha, respectivement à la tête du quotidien genevois La Suisse, de la Correspondance politique suisse et de la Basler Zeitung. Leur projet? Lancer la première télévision suisse par satellite. L'ambitieuse initiative tombera à l'eau. Mais sa courte histoire n'en demeure pas moins significative tant elle agrège les enjeux politiques, économiques et culturels qui agitent le monde médiatique helvétique de ce début de décennie.

Tout d'abord, Tel-Sat s'oppose de front au modèle d'une télévision de service public, et ceci dans un contexte de forte pression contre le statut privilégié de la Société suisse de radiodiffusion (SSR). Celle qui détient depuis sa création le monopole sur les ondes subit en effet des attaques croissantes à partir du milieu des années 1970. Or, la demande de concession déposée par Tel-Sat pour obtenir le droit de diffuser des programmes remet en cause, de fait, ce monopole tant décrié. Et les caractéristiques du projet défient plus encore l'organisation traditionnelle du petit écran en Suisse. À rebours d'un financement par la redevance, ses promoteurs tablent sur la publicité. À la gestion gouvernementale et nationale, les actionnaires préfèrent s'associer aux puissants industriels britanniques Thorn-EMI et British Aerospace qui doivent fournir le savoir-faire et le matériel pour construire le satellite. Finalement, alors que les émissions de la SSR sont destinées aux 
citoyens et citoyennes helvétiques, l'initiative commerciale ambitionne d'atteindre une audience internationale que convoitent déjà plusieurs concurrents.

Lors d'une conférence de presse, Jean-Claude Nicole énumère les nombreux plans de télévision satellite qui s'élaborent dans les nations voisines et demande:

Dans cette évolution, notre pays sera-t-il présent ou absent, actif ou passif? [...] Sachant que des programmes de télévision avec publicité nous viendront de l'espace, voulons-nous ou non un satellite suisse?

Pour le directeur de La Suisse, la réponse est évidemment oui, et le plus vite possible: il s'agit d'être les premiers à conquérir les parts du marché publicitaire. En bref, Tel-Sat constitue l'antithèse radicale d'une télévision monopolistique et de service public. Selon une édition du Journal de Genève d'avril 1980, elle a de quoi «faire trembler la SSR».

La nature commerciale de l'entreprise alimente donc les discussions sur l'avenir du paysage audiovisuel en Suisse. Mais pas seulement: le statut de «nouveau média» associé à la télévision satellite détermine également la manière dont Tel-Sat s'insère dans le débat public. À l'orée de la décennie 1980, plusieurs nouvelles technologies viennent en effet redéfinir l'écologie médiatique du pays. Avec la télévision par câble, le télétexte, le vidéotex ou la télématique, la radiodiffusion par satellite suscite les craintes et l'enthousiasme qui accompagnent généralement l'émergence de ce qu'on appelle désormais des «nouveaux médias». L'usage de l'expression, apparue au milieu du siècle, connaît une croissance importante à cette époque et vient ainsi empreindre de son aura révolutionnaire le développement de l'électronique. En témoigne une série de chroniques publiées entre mai et juin 1980 dans L'Express sous le titre «Tel-Sat, Videotex, Teletel, etc.». Pour le journaliste, ces «fameux nouveaux média[s]» n'inaugurent pas moins qu'une "nouvelle ère de civilisation" dont il redoute les "conséquences incalculables" sur la qualité de vie en même temps qu'il se réjouit de "l'extension des connaissances et du savoir». La télévision satellite annonce quant à elle l'augmentation du choix de programmes, mais aussi «l'entrée simultanée [de la publicité de source internationale] dans les foyers helvétiques", alors que "la notion même de frontière deviendra plus floue».

Cette peur d'un déferlement incontrôlable de contenus étrangers, faisant fi des règlementations ou de la culture locales, accompagne 
l'évolution de la télévision satellite dès les premières expériences de Mondovision dans les années 1960. Toutefois, en ce début de décennie 1980, la question se pose avec une acuité nouvelle en Europe; la fusée Ariane a effectué son vol inaugural en 1979, l'Agence spatiale européenne est désormais capable d'envoyer des satellites sur orbite. Par ailleurs, la technique a progressé. Les signaux sont à présent suffisamment puissants pour être captés par des antennes de taille moyenne à petite, soit par des infrastructures moins coûteuses et accessibles à un plus grand nombre. Des programmes réguliers peuvent être envisagés. Le temps de la télévision qui vient "directement du ciel» semble proche.

Ces évolutions dans le domaine médiatique font réagir ses institutions bien établies, notamment les grands titres de presse. En avril 1980, l'Association suisse des éditeurs de journaux (ASEJ) accorde ainsi la priorité «à son action dans le domaine des nouveaux moyens et des nouvelles techniques d'information», se voulant «résolument tournée vers l'avenir». Deux ans plus tard, la thématique occupe les discussions de son Assemblée générale à Baden. À cette occasion, son président Max Rapold invite ses condisciples à "ne pas rester à l'écart de ce qu’on appelle les nouveaux médias» et les enjoint au contraire à "participer à leur mise sur pied». Cette stratégie a été prise au mot par les promoteurs de TelSat. En avril 1980, Jean-Claude Nicole présente l'entreprise lors d'une réunion de l'ASEJ où il espère convaincre de nouveaux partenaires. Il a cette phrase:

[P]our la première fois, la presse suisse a la possibilité de prendre directement en mains un nouveau média, dont on sait qu'à défaut d'être exploité par elle, il le sera par d'autres.

Tel-Sat ne connaîtra pas le succès escompté. Après quatre longues années de négociations, le Conseil fédéral refuse l'octroi d'une concession. Le projet révèle néanmoins comment la presse s'efforce de s'adapter en période d'incertitudes ou de transformations. Son histoire pointe aussi la difficulté de tracer des frontières claires entre les différents médias qui apparaissent en fait en constante interaction. Les éditeurs de journaux participeront d'ailleurs aux premiers essais de Télétexte en 1981 et de Vidéotex en 1983, en partenariat avec la SSR.

Le monopole de cette dernière tombe en 1984, à une époque où, en Europe occidentale, libéralisation de l'économie rime avec celle de l'audiovisuel. La télévision nationale demeure toutefois dominante. En 
matière de diffusion satellitaire, la SSR crée des alliances stratégiques dont elle cueille les premiers fruits l'année même de l'abolition de son statut: en janvier, la collaboration avec d'autres radiodiffuseurs de service public aboutit au lancement de la chaine francophone TV5; en mai, un accord avec le secteur privé débouche sur les premières diffusions de Teleclub, télévision suisse par abonnement et via satellite. En définitive, l'aventure de Tel-Sat est un saisissant révélateur des reconfigurations et des luttes à l'œuvre dans le domaine des médias en cette année 1980. Des changements techniques à leurs perceptions culturelles en passant par la recomposition des équilibres entre les multiples acteurs en jeu.

Marie SANDOZ Section d'histoire, Faculté des lettres, Université de Lausanne

\section{BIBLIOGRAPHIE}

Archives cantonales vaudoises: PP 888 101, Télévision par satellite (1980-1987) et PP 888/99, Télétexte et Vidéotex (1978-1986).

L'Express, 23, 24, 27, 30 et 31 mai; 1 1 , 2, 3, 4, 5 et 6 juin 1980.

Mäusli, Theo, Steigmeier, Andreas, Vallotton, François (éds), La radio et la télévision en Suisse: histoire de la Société suisse de radiodiffusion et télévision SSR de 1983 à 2011, Baden, hier + jetzt, 2012. 